\section{Determinantes biológicos e sócio-culturais associados à prática de atividade física de adolescentes}

\author{
Biological and socio-cultural determinants of \\ physical activity in adolescents
}

\footnotetext{
${ }^{1}$ Faculdade de Desporto, Universidade do Porto, Porto, Portugal.

2 Instituto de Ciências Biomédicas Abel Salazar. Universidade do Porto, Porto, Portugal. 3 Faculty of Kinesiology and Rehabilitation Sciences, Katholieke Universiteit Leuven, Leuven, Belgium. 4 Departamento de Nutrição Social, Universidade Federa Fluminense, Niterói, Brasil.

${ }_{5}$ Escola Nacional de Saúde Pública Sergio Arouca, Fundação Oswaldo Cruz, Rio de Janeiro, Brasil.

Correspondência A. F. Seabra Laboratório de Cineantropometria e Estatística Aplicada, Faculdade de Desporto, Universidade do Porto. Rua Dr. Plácido Costa 91, Porto 4200-450, Portugal. aseabra@fade.up.pt
}

\begin{abstract}
Physical activity is important for health promotion and disease prevention. Effective physical activity programs for adolescents require a proper understanding of the determinants of activity levels. The main purpose of this paper was to review the scientific literature on determinants of physical activity among adolescents: demographic, biological (age, gender, socioeconomic status), and socio-cultural (family, peers, and physical education teachers). The review included only studies with large samples $(\geq 100$ subjects) and a cross-sectional design, and that used questionnaires to measure physical activity in adolescents (10-18 years). The main results and conclusions were: age is negatively associated with physical activity; boys tend to be more active than girls; higher socioeconomic status is positively associated with more physical activity; adolescents are more involved in physical activity when parents and peers also participate; physical education teachers do not influence the adolescents' level of physical activity.
\end{abstract}

Motor Activity; Adolescent; Adolescente Behavior

\author{
André F. Seabra 1 \\ Denisa M. Mendonça 2 \\ Martine A. Thomis 3 \\ Luiz A. Anjos 4,5 \\ José A. Maia 1
}

\section{Introdução}

A atualidade é percorrida por um forte incremento da morbidade associada a doenças não infecciosas e crônico-degenerativas que se pensa estar na dependência estreita da drástica alteração no estilo de vida das populações. Desde os tempos em que era caçador-recoletor até o presente, o homem modificou substancialmente a sua forma de estar e viver, sobretudo nos últimos 100-150 anos com a revolução industrial e mais recentemente com a robótica. Passou a ser mais inativo, a consumir mais tabaco e a aderir a hábitos nutricionais cada vez menos saudáveis.

Não é pois de estranhar que a Organização Mundial da Saúde (OMS) 1 tenha referido que cerca de $60 \%-85 \%$ da população dos países desenvolvidos e dos países em transição tenham estilos de vida sedentários. Foi estimado que, em todo o mundo, a estimativa global da prevalência de inatividade física em indivíduos com idade superior a 15 anos é de $17 \%$, variando entre os $11 \%$ e os $24 \%$ consoante as regiões. Num outro estudo conduzido por Sjostrom et al. ${ }^{2}$ em países da Comunidade Européia, 31\% dos sujeitos com idade superior a 15 anos foram considerados sedentários. Também no Brasil, recente revisão da situação no país indicou prevalências que variaram de $26,7 \%$ a $78,2 \%$, dependendo da região e da faixa etária estudada 3 .

Este quadro alarmante obrigou a que organizações científico-médicas tenham declarado 
o sedentarismo e a inatividade física como um dos maiores problemas de Saúde Pública das sociedades modernas. Tão grande é esta epidemia, que um número cada vez maior de adolescentes está afetado por este comportamento de risco. De fato, uma pesquisa desenvolvida pela OMS 4 em adolescentes com idade inferior a 15 anos salientou que dois terços desses adolescentes não cumpriam as linhas de recomendação da atividade física, isto é, uma hora ou mais de atividade, a uma intensidade no mínimo moderada, em cinco ou mais dias da semana. Igualmente no Brasil, o estudo de Hallal et al. 5 em adolescentes de 10-12 anos de idade mostrou prevalências de sedentarismo (menos de 300 minutos de atividade física por semana) de 58\%. Tais resultados são preocupantes na medida em que os hábitos de atividade física regular na adolescência parecem ter efeitos benéficos em alguns fatores de risco e em algumas doenças crônicas não transmissíveis 6,7 bem como tendem a repercutir-se no estado adulto dos sujeitos 8 .

Decorre daqui a necessidade de se estabelecerem estratégias e programas de intervenção que visem promover, junto dos adolescentes, estilos de vida ativos e saudáveis fazendo da ativi- dade física uma parte muito importante. Parece ser claro que a eficácia de tais programas depende da identificação e modificação de aspectos e de fatores que determinam a participação nessas atividades 9,10 .

Da análise crítica da literatura emerge a noção de não haver ainda uma descrição esclarecedora dos múltiplos fatores que determinam a forte variabilidade nos hábitos de atividade física das populações infanto-juvenis. O quadro conceptual disponível, de acordo com Sallis 11 e Bauman et al. 12 é um conjunto díspar, nem sempre convergente de autodesignadas "teorias" e modelos que têm orientado a investigação e que originam um lote de variáveis correlatas (i.e., determinantes) que se pensa influenciar aspectos da complexidade comportamental associada à atividade física (Tabela 1).

É reconhecimento unânime dessa multiplicidade de abordagens do problema da interpretação da forte variabilidade populacional, nos níveis de atividade física, que este tipo de atividade é um comportamento extremamente complexo e multifatorial 13. Decorre daqui que é impensável atribuir a qualquer um desses determinantes a maior fatia da variância total observada e que a

"Teorias" e modelos utilizados na investigação da influência de fatores determinantes dos hábitos de atividade física (adaptado de Sallis \& Owen 42).

\begin{tabular}{|c|c|c|c|c|}
\hline "Teoria"/Modelo & $\begin{array}{l}\text { Variáveis } \\
\text { intrapessoais }\end{array}$ & $\begin{array}{l}\text { Variáveis } \\
\text { sociais }\end{array}$ & $\begin{array}{l}\text { Variáveis de } \\
\text { envolvimento físico }\end{array}$ & $\begin{array}{l}\text { Aplicações e } \\
\text { intervenções }\end{array}$ \\
\hline Crença na saúde & $\begin{array}{c}\text { Percepção na suscetibilidade, } \\
\text { na severidade, nos } \\
\text { benefícios e nas barreiras; } \\
\text { auto-eficácia; disposição } \\
\text { para ação }\end{array}$ & - & - & $\begin{array}{l}\text { Programas baseados } \\
\text { no conhecimento; } \\
\text { educação para } \\
\text { a saúde; avaliação } \\
\text { do risco }\end{array}$ \\
\hline $\begin{array}{l}\text { Comportamento } \\
\text { planejado }\end{array}$ & $\begin{array}{l}\text { Intenções comportamentais; } \\
\text { atitude perante o } \\
\text { comportamento; percepção } \\
\text { do controlo do comportamento }\end{array}$ & $\begin{array}{c}\text { Normas subjetivas: } \\
\text { percepções das crenças } \\
\text { dos outros e motivação } \\
\text { para o consentimento }\end{array}$ & - & $\begin{array}{l}\text { Mudança de atitude } \\
\text { na comunicação }\end{array}$ \\
\hline Trans-teórico & $\begin{array}{c}\text { Mudança de estádios e } \\
\text { de processos; auto-eficácia }\end{array}$ & $\begin{array}{l}\text { Alguns processos de } \\
\text { mudança; algumas } \\
\text { variáveis de decisão }\end{array}$ & $\begin{array}{l}\text { Alguns processos de } \\
\text { mudança; algumas } \\
\text { variáveis de decisão }\end{array}$ & $\begin{array}{c}\text { Modificação cognitiva } \\
\text { e comportamental }\end{array}$ \\
\hline Sócio-cognitiva & $\begin{array}{c}\text { Resultados esperados; } \\
\text { capacidade comportamental; } \\
\text { auto-eficácia }\end{array}$ & $\begin{array}{l}\text { Aprendizagem por } \\
\text { observação; reforço }\end{array}$ & Reforço & $\begin{array}{l}\text { Modificação do } \\
\text { comportamento } \\
\text { cognitivo }\end{array}$ \\
\hline Ecológico & $\begin{array}{l}\text { Múltiplos níveis de } \\
\text { influência, incluindo o } \\
\text { intrapessoal }\end{array}$ & $\begin{array}{c}\text { Fatores interpessoais } \\
\text { e institucionais }\end{array}$ & $\begin{array}{c}\text { Fatores comunitários e } \\
\text { de política pública; } \\
\text { envolvimentos promotores } \\
\text { de saúde }\end{array}$ & Abordagem multiníve \\
\hline
\end{tabular}


importância relativa dos determinantes permanece invariante ao longo da idade e nos dois sexos 14 . Não obstante essa consideração, Buckworth \& Dishman 15 referem genericamente, sem qualquer esforço de atribuição de magnitudes de efeito, que os fatores que determinam a atividade física podem ser categorizados em atributos pessoais do passado e do presente, fatores do envolvimento do passado e do presente, e aspectos da própria atividade física. Por um posicionamento operativo e sistemático, é possível distribuir com algum rigor o conjunto diversificado de fatores e determinantes que parecem influenciar a atividade física de adolescentes (Tabela 2).

É consensual que aspectos demográfico-biológicos (idade, sexo, estatuto sócio-econômico), psicológicos (motivação) e sócio-culturais (família e pares) influenciam a heterogeneidade populacional nos hábitos de atividade física em adolescentes. O problema principal reside na hierarquia da sua importância. Em razão disso, nas últimas décadas, diferentes linhas de pesquisa têm tentado identificar, dentre os vários fatores e determinantes previamente mencionados, aqueles que mais influenciavam a atividade física de adolescentes (ver Dishman et al. 9 e Sallis et al. 16). No entanto, a possibilidade de sumariar os resultados provenientes desses estudos tem sido uma tarefa complexa pelos seguintes motivos.

- Ausência de distinção clara entre os conceitos atividade física e exercício físico, uma vez que são freqüentemente utilizados como sinônimos na literatura. Apesar de estarem inter-relacionados, refletem estruturas conceituais e operativas distintas. A atividade física é consensualmente entendida como qualquer movimento corpo- ral produzido pelos músculos esqueléticos que resulta num aumento do dispêndio energético relativamente à taxa metabólica de repouso. $\mathrm{O}$ exercício físico, por seu lado, é entendido como uma subcategoria da atividade física, sendo habitualmente descrito como todo o movimento corporal planejado, estruturado e repetitivo, que resulta na manutenção ou melhoria de uma ou mais componentes da aptidão física 10,17.

- Utilização de diferentes delineamentos de pesquisa, o que conduz a resultados e a interpretações distintas. Dishman et al. 18 referem que os delineamentos transversais são os que mais se ajustam às pesquisas epidemiológicas da atividade física pela maior facilidade em estudarem grandes amostras, pela rapidez de execução e pelo baixo custo. Contudo, esses delineamentos apenas permitem conhecer o padrão de distribuição da atividade num dado ponto do tempo e sugerir hipóteses sobre possíveis associações entre a atividade física e determinados fatores de risco de algumas doenças. Para o conhecimento suficientemente esclarecedor acerca da história natural da atividade física no decurso do tempo, ter-se-ia que utilizar um delineamento longitudinal. Todavia, apesar das suas vantagens, esse tipo de delineamento também possui algumas limitações, nomeadamente: a dificuldade de utilização em grandes amostras, a complexidade operativa, a "morte" amostral, a morosidade na obtenção de resultados e os custos necessários à sua realização 19 .

- Dimensões amostrais diversas, o que condiciona todas as inferências que se possam realizar. Na literatura, observam-se estudos cujo número de participantes não ultrapassa uma centena e outros em que é de alguns milhares de sujeitos.

Fatores Determinantes

Demográficos e biológicos

Psicológicos, emocionais e cognitivos

Comportamentais

Sócio-culturais

Ambientais

Características da atividade física
Idade; habilitações acadêmicas; sexo; genética; estatuto sócio-econômico; características físicas antropométricas/composição corporal; etnia

Gosto pelos exercícios; alcance de benefícios; desejo de exercitar-se; distúrbios do humor; percepção de saúde e aptidão; senso pessoal de competência; motivação

História de atividade anterior; qualidade dos hábitos dietéticos; processos de mudança Influência do médico; apoio social dos amigos/pares; apoio social da família; apoio social dos professores

Acesso a equipamentos (percepção); clima; custos dos programas; interrupção da rotina Intensidade; sensação subjetiva do esforço 
Essa dispersão no tamanho amostral dificulta as inferências e generalizações possíveis de serem feitas sobre determinados parâmetros da população 14 .

- Diversidade de instrumentos utilizados para avaliar a atividade física 20,21,22. Existem estudos que utilizaram instrumentos de natureza laboratorial (calorimetria e marcadores fisiológicos) e de terreno (sensores de movimento, diários, questionários). Uma vez que cada um destes instrumentos mede diferentes facetas da atividade física, conduzem naturalmente a resultados diferenciados.

Apesar do conjunto de problemas inventariados, o presente trabalho objetiva rever alguns aspectos do estado atual do conhecimento acerca da influência de determinantes de âmbito demográfico-biológicos (idade, sexo, estatuto sócio-econômico) e sócio-cultural (família, pares e professor de educação física) na atividade física de adolescentes.

\section{Métodos}

Face ao quadro atual de constrangimentos existente e para que o presente estudo pudesse concretizar os seus propósitos, houve a necessidade de definir os seguintes critérios de inclusão dos estudos a revisar: (1) que tenham abordado a atividade no seu conceito mais amplo 17; (2) que tenham analisado a influência de determinan- tes demográfico-biológicos (idade, sexo, estatuto sócio-econômico) e sócio-culturais (família, pares e professor de educação física) na atividade física de adolescentes; (3) que tenham sido realizados com amostras de dimensão superior a 100 adolescentes com idades compreendidas entre os 10 e os 18 anos; (4) dada a escassez de estudos epidemiológicos de âmbito longitudinal 23 apenas selecionar estudos que tenham adotado delineamentos de pesquisa transversal; e (5) que tenham utilizado questionários como instrumento de avaliação da atividade física independentemente do período de recordação a que se referem e das unidades de medida que estimam. A opção pela utilização deste instrumento resulta do número significativamente superior de estudos existentes na literatura comparativamente a pesquisas que adotaram outro instrumento.

Para a pesquisa dos artigos foram consultadas as bases de dados MEDLINE e SportDiscus, entre 1977 e 2006, utilizando-se as palavras-chave em inglês: "physical activity", "sport participation", "demographic-biological AND social-cultural determinants", "adolescents".

\section{Resultados}

A Tabela 3 lista as 41 investigações epidemiológicas que foram selecionadas por cumprirem todos os critérios anteriores.

Autor, ano de realização, dimensão da amostra, país, tipo de questionário e determinantes utilizados em pesquisas sobre atividade física em adolescentes.

\begin{tabular}{|c|c|c|c|c|c|c|c|}
\hline Autor & $\begin{array}{c}\text { Ano da } \\
\text { realização }\end{array}$ & $\mathbf{n}$ & $\begin{array}{l}\text { Amostra } \\
\text { Idade } \\
\text { (anos) }\end{array}$ & $\begin{array}{c}\text { Escolaridade } \\
\text { (anos) }\end{array}$ & País & $\begin{array}{c}\text { Tipo de } \\
\text { questionário }\end{array}$ & $\begin{array}{c}\text { Fatores } \\
\text { determinantes }\end{array}$ \\
\hline Andersen \& Wold 75 & 1992 & 904 & 13 & - & Noruega & $\mathrm{NI}$ & Sócio-culturais \\
\hline Andújar \& Piéron 44 & 2000 & 700 & $12-16$ & - & Espanha & $\mathrm{NI}$ & Sócio-culturais \\
\hline Bungun et al. 45 & 2000 & 520 & $13-19$ & - & Estados Unidos & PDPAR & $\begin{array}{c}\text { Psicológicos, emocionais } \\
\text { e cognitivos }\end{array}$ \\
\hline Cleland et al. 85 & 2005 & 5.929 & $9-15$ & - & Austrália & $\mathrm{NI}$ & $\begin{array}{c}\text { Demográficos e } \\
\text { biológicos; sócio-culturais }\end{array}$ \\
\hline Duncan et al. 52 & 2002 & 301 & $12-14$ & - & Inglaterra & FODRQ & $\begin{array}{c}\text { Demográficos e } \\
\text { biológicos }\end{array}$ \\
\hline Duncan et al. 67 & 2005 & 372 & 12 & - & Estados Unidos & $\mathrm{NI}$ & $\begin{array}{c}\text { Demográficos e } \\
\text { biológicos; sócio-culturais }\end{array}$ \\
\hline Fogelholm et al. 86 & 1999 & 271 & 10 & - & Finlândia & 3DPAR & Sócio-culturais \\
\hline Godin \& Shephard 80 & 1986 & 698 & - & $7-8$ & Canadá & GSPAS & $\begin{array}{l}\text { Psicológicos, emocionais } \\
\text { e cognitivos; sócio-culturais }\end{array}$ \\
\hline
\end{tabular}

(continua) 
Tabela 3 (continuação)

\begin{tabular}{|c|c|c|c|c|c|c|c|}
\hline Autor & $\begin{array}{l}\text { Ano da } \\
\text { realização }\end{array}$ & $\mathbf{n}$ & $\begin{array}{l}\text { Amostra } \\
\text { Idade } \\
\text { (anos) }\end{array}$ & $\begin{array}{l}\text { Escolaridade } \\
\text { (anos) }\end{array}$ & País & $\begin{array}{c}\text { Tipo de } \\
\text { questionário }\end{array}$ & $\begin{array}{c}\text { Fatores } \\
\text { determinantes }\end{array}$ \\
\hline Gordon-Larsen et al. 59 & 2000 & 17.766 & - & $7-12$ & Estados Unidos & $\mathrm{NI}$ & $\begin{array}{c}\text { Demográficos e biológicos; } \\
\text { sócio-culturais }\end{array}$ \\
\hline Gottlieb \& Chen 87 & 1985 & 2.695 & - & $7-8$ & Estados Unidos & $\mathrm{NI}$ & $\begin{array}{c}\text { Demográficos e biológicos; } \\
\text { sócio-culturais }\end{array}$ \\
\hline Greendorfer 79 & 1977 & 585 & $5-22$ & - & Estados Unidos & $\mathrm{NI}$ & Sócio-culturais \\
\hline Gregson \& Colley 88 & 1986 & 236 & $15-16$ & - & Inglaterra & $\mathrm{NI}$ & Sócio-culturais \\
\hline Hallal et al. 5 & 2006 & 4.451 & $10-12$ & - & Brasil & $\mathrm{NI}$ & Demográficos e biológicos \\
\hline Kristjansdottir \& & 2001 & 3.270 & $11-16$ & - & Islândia & $\mathrm{NI}$ & Demográficos e biológicos \\
\hline Vilhjalmsson 25 & & & & & & & \\
\hline Lasheras et al. 36 & 2001 & 1.358 & $6-15$ & - & Espanha & $\mathrm{NI}$ & Demográficos e biológicos \\
\hline Lindquist et al. 89 & 1999 & 107 & $7-13$ & - & Estados Unidos & MKAQ & $\begin{array}{c}\text { Demográficos e biológicos; } \\
\text { sócio-culturais }\end{array}$ \\
\hline Mota \& Silva 62 & 1999 & 498 & - & $7-9$ & Portugal & AWAC & Demográficos e biológicos \\
\hline Oehlschlaeger et al. 46 & 2004 & 960 & $15-18$ & - & Brasil & $\mathrm{NI}$ & Demográficos e biológicos \\
\hline O'Loughlin et al. 26 & 1999 & 2.285 & $9-13$ & - & Canadá & AWAC & $\begin{array}{c}\text { Demográficos e biológicos; } \\
\text { psicológicos, emocionais e } \\
\text { cognitivos; sócio-culturais }\end{array}$ \\
\hline Pate et al. 27 & 1997 & 361 & - & 5 & Estados Unidos & PDPAR & $\begin{array}{l}\text { Demográficos e biológicos; } \\
\text { psicológicos, emocionais e } \\
\text { cognitivos; sócio-culturais }\end{array}$ \\
\hline Pereira 28 & 1999 & 517 & $12-19$ & - & Portugal & $\mathrm{BO}$ & $\begin{array}{c}\text { Demográficos e biológicos; } \\
\text { sócio-culturais }\end{array}$ \\
\hline Pizarro \& Sherrill 77 & 1991 & 447 & $9-12$ & - & Costa Rica & SII & Sócio-culturais \\
\hline Raudsepp \& Viira 72 & 2000 & 475 & $13-15$ & - & Estônia & 7DPAR & Sócio-culturais \\
\hline Raudsepp \& Viira 73 & 2000 & 375 & $13-14$ & - & Estônia & 7DPAR & Sócio-culturais \\
\hline Rossow \& Rise 43 & 1994 & 337 & $16-20$ & - & Noruega & $\mathrm{NI}$ & Sócio-culturais \\
\hline Sallis et al. 47 & 1996 & 1.871 & $11-19$ & - & Estados Unidos & NHIS & $\begin{array}{c}\text { Demográficos e biológicos; } \\
\text { psicológicos, emocionais e } \\
\text { cognitivos; sócio-culturais }\end{array}$ \\
\hline Sallis et al. 65 & 1999 & 1.504 & - & $4-12$ & Estados Unidos & 1DR & $\begin{array}{c}\text { Demográficos e biológicos; } \\
\text { psicológicos, emocionais e } \\
\text { cognitivos; sócio-culturais }\end{array}$ \\
\hline Schmitz et al. 37 & 2002 & 3.798 & $11-15$ & - & Estados Unidos & $\mathrm{NI}$ & Demográficos e biológicos \\
\hline Seabra 38 & 2004 & 5.850 & $10-18$ & - & Portugal & BO & $\begin{array}{c}\text { Demográficos e biológicos; } \\
\text { sócio-culturais }\end{array}$ \\
\hline Shropshire \& Carroll 63 & 1997 & 924 & - & 6 & Inglaterra & $\mathrm{NI}$ & $\begin{array}{c}\text { Demográficos e biológicos; } \\
\text { sócio-culturais }\end{array}$ \\
\hline $\begin{array}{l}\text { Stucky-Roop \& } \\
\text { DiLorenzo } 78\end{array}$ & 1993 & 242 & - & $5-6$ & Estados Unidos & $\mathrm{NI}$ & $\begin{array}{l}\text { Demográficos e biológicos; } \\
\text { psicológicos, emocionais e } \\
\text { cognitivos; sócio-culturais }\end{array}$ \\
\hline Surís \& Parera 29 & 2005 & 6.928 & $14-19$ & - & Espanha & $\mathrm{NI}$ & $\begin{array}{c}\text { Demográficos e biológicos; } \\
\text { sócio-culturais }\end{array}$ \\
\hline Trost et al. 48 & 1996 & 334 & - & 5 & Estados Unidos & PDPAR & $\begin{array}{c}\text { Demográficos e biológicos; } \\
\text { psicológicos, emocionais e } \\
\text { cognitivos }\end{array}$ \\
\hline $\begin{array}{l}\text { Vilhjalmsson \& } \\
\text { Thorlindsson } 49\end{array}$ & 1998 & 1.131 & $15-16$ & - & Islândia & $\mathrm{NI}$ & $\begin{array}{l}\text { Demográficos e biológicos; } \\
\text { psicológicos, emocionais e } \\
\text { cognitivos; sócio-culturais }\end{array}$ \\
\hline $\begin{array}{l}\text { Vilhjalmsson \& } \\
\text { Kristjansdottir } 30\end{array}$ & 2003 & 3.270 & - & $6-10$ & Islândia & $\mathrm{NI}$ & $\begin{array}{c}\text { Demográficos e biológicos; } \\
\text { sócio-culturais }\end{array}$ \\
\hline Wagner et al. 50 & 2002 & 3.437 & - & 6 & França & MAQA & $\begin{array}{c}\text { Demográficos e biológicos; } \\
\text { sócio-culturais }\end{array}$ \\
\hline
\end{tabular}

(continua) 


\begin{tabular}{|c|c|c|c|c|c|c|c|}
\hline \multirow[t]{2}{*}{ Autor } & \multirow{2}{*}{$\begin{array}{c}\text { Ano da } \\
\text { realização }\end{array}$} & \multicolumn{3}{|c|}{ Amostra } & \multirow[t]{2}{*}{ País } & \multirow{2}{*}{$\begin{array}{c}\text { Tipo de } \\
\text { questionário }\end{array}$} & \multirow{2}{*}{$\begin{array}{c}\text { Fatores } \\
\text { determinantes }\end{array}$} \\
\hline & & $\mathrm{n}$ & $\begin{array}{l}\text { Idade } \\
\text { (anos) }\end{array}$ & $\begin{array}{c}\text { Escolaridade } \\
\text { (anos) }\end{array}$ & & & \\
\hline Wold \& Andersen 74 & 1992 & 39.086 & $11-16$ & - & Países europeus & $\mathrm{NI}$ & $\begin{array}{l}\text { Demográficos e biológicos; } \\
\text { psicológicos, emocionais e } \\
\text { cognitivos; sócio-culturais }\end{array}$ \\
\hline Wolf et al. 31 & 1993 & 552 & - & $5-12$ & Estados Unidos & GSPAS & Demográficos e biológicos \\
\hline Woodfield et al. 34 & 2002 & 301 & 13 & - & Inglaterra & FODRQ & Demográficos e biológicos \\
\hline Yang et al. 64 & 1996 & 1.881 & $9-15$ & - & Finlândia & $\mathrm{NI}$ & $\begin{array}{c}\text { Demográficos e biológicos; } \\
\text { sócio-culturais }\end{array}$ \\
\hline Zakarian et al. 32 & 1994 & 1.634 & - & $9-12$ & Estados Unidos & $\mathrm{NI}$ & $\begin{array}{l}\text { Demográficos e biológicos; } \\
\text { psicológicos, emocionais e } \\
\text { cognitivos; sócio-culturais }\end{array}$ \\
\hline
\end{tabular}

NI: não identificado; BQ: Baecke Questionnaire; PDPAR: Previous Day Physical Activity Recall; FODRQ: Four by One Day Recall Questionnaire; AWAC: Adaptation of the Weekly Activity Checklist; SII: Sport Interest Inventory; 3DPAR: 3-Day Physical Activity Record; 7DPAR: 7-Day Physical Activity Record; NHIS: National Health Interview Survey; GSPAS: Godin-Shephard Physical Activity Survey; MKAQ: Modified Kriska Activity Questionnaire; 1DR: One Day Recall; MAQA: Modifiable Activity Questionnaire for Adolescents.

Pela sua análise, observam-se pesquisas cujo número de participantes não ultrapassa uma centena e outros em que esse número é superior a muitos milhares de adolescentes (mínimo: 107; máximo: 39.086). Também se verifica uma enorme variação na distribuição dos estudos pelos diferentes continentes (20 na Europa; 17 na América do Norte; 2 na América do Sul; 1 na América Central; 1 na Oceania) e no tipo de questionário utilizado para avaliar a atividade física (15 tipos diferentes).

\section{Determinantes demográfico-biológicos}

Segundo Carron et al. 24, os determinantes demográfico-biológicos não são passíveis de serem alterados na data de recolhimento da informação por causa do caráter transversal, e incluem normalmente a idade, a etnia, o estatuto sócio-econômico e o sexo. Neste trabalho, apenas centraremos a nossa atenção na idade, no sexo e no estatuto sócio-econômico, por serem fatores muito estudados e por mostrarem alguma influência na atividade física de adolescentes.

\section{Idade}

Na literatura consultada, a idade é considerada um importante determinante da atividade física de adolescentes. Apesar de algumas diferenças conforme o tipo e a intensidade da atividade física realizada, é consensual o entendimento de que a atividade física, compreendida no seu conceito global, tende a estar negativamente associada à idade. Parece ser evidente que as atividades desportivas e de intensidade vigorosa são as que preferencialmente diminuem com o avanço da idade 25,26,27,28,29,30,31,32. Porém, há estudos que não mostraram esse tipo de associação. Pesquisas epidemiológicas descritivas 33,34,35 e analíticas 36,37,38 salientaram um declínio com o decorrer da idade das atividades de lazer de intensidade moderada a vigorosa e um aumento das atividades de baixa intensidade.

Embora diversos autores tenham procurado identificar as razões para esse declínio, permanece por esclarecer se é resultado de fatores biológicos ou ligados ao envolvimento, se da sua interação ou se por qualquer outro mecanismo de natureza social que tenha escapado ao olhar inquisitivo dos pesquisadores.

É possível encontrar autores 39,40,41 que consideram os fatores biológicos como responsáveis por esse declínio. Rowland 39 sugeriu a existência, no sistema nervoso central, de um centro de controle com capacidade para governar a atividade física regular. De acordo com este autor, a hiperatividade das crianças e a natureza das suas brincadeiras suportam a idéia de que a atividade é fortemente controlada por fatores biológicos. Tal atividade é vista em todo o reino animal, especialmente nos mais jovens, como uma forma de manter "desperta" a maior parte das atividades do sistema nervoso central.

Também Thorburn \& Proietto 40 procuraram mostrar, em humanos e animais, a existência de mecanismos de controle biológico dos níveis de atividade física espontânea. Dentre os diversos fatores com capacidade para influenciar negativamente a atividade física espontânea com o 
decurso da idade, destaca-se a insuficiência de estrogênio e as baixas doses de noradrenalina, serotonina e dopamina.

Ingram ${ }^{41}$, numa pesquisa realizada com animais, reforça alguns dos resultados anteriores. O autor identifica a menor libertação da dopamina com o avanço da idade como o principal fator responsável pela diminuição nos níveis de atividade física com a idade, já que a dopamina está essencialmente associada à motivação para a realização de movimento, atuando em regiões específicas do cérebro.

Para além de aspectos biológicos, existem outros de natureza não biológica, nomeadamente fatores sociais, culturais e ligados ao envolvimento, com responsabilidade na diminuição dos níveis de atividade física com a idade 9,42,43. Um dos fatores de âmbito social que poderá ajudar a compreender o declínio da atividade física é a alteração que se verifica ao longo da idade nos modelos que orientam os comportamentos dos adolescentes. Enquanto na infância a família parece ser o primeiro e o mais poderoso agente socializador na transmissão de valores, comportamentos e normas, a entrada na adolescência leva os adolescentes a desenvolver um sentido de autonomia e de independência que os motiva, na grande maioria das vezes, a "separarem-se" dos seus pais. Essa "separação" traduz-se geralmente numa alteração dos seus modelos e valores, fato que conduz a uma transferência das influências da família para os pares, o que, por sua vez, pode influenciar os comportamentos e os estilos de vida saudáveis que o adolescente evidenciava até então. Um outro aspecto do envolvimento social nessa fase é a escola e, mais especificamente, a disciplina de educação física e o seu professor. Efetivamente, os adolescentes passam diariamente uma grande parte do seu tempo na escola, sofrendo diversas influências positivas e/ou negativas que poderão condicionar os seus hábitos de atividade física.

Em suma, apesar de algumas divergências de acordo com o tipo e a intensidade da atividade física realizada, existe uma certa unanimidade da presença de um declínio da atividade física com o avanço da idade. Perante esses resultados é fundamental o estabelecimento de programas de intervenção, altamente motivadores, diversificados e indutores de sucesso na alteração dos estilos de vida dos adolescentes, sobretudo programas culturalmente referenciados e gratificantes que envolvam também os outros significantes (por exemplo: família, pares, escola, professores, profissionais ligados à saúde etc.).

\section{Sexo}

A grande maioria dos estudos realizados tem mostrado que o sexo masculino é fisicamente mais ativo que o feminino 11,25,26,27,29,36,38,44,45, $46,47,48,49,50$. As diferenças que se registram entre sexos estão algo dependentes do tipo e da intensidade da atividade física realizada. Diversos estudos epidemiológicos descritivos, reconhecem um envolvimento superior dos meninos em atividades físicas de natureza desportiva e de intensidade vigorosa 51,52 , contrariamente às meninas que parecem mostrar prevalências superiores de participação em atividades físicas de lazer e de baixa intensidade 34,53 .

As razões para as diferenças na participação em atividades físicas de tipo e intensidade variados não são claras. Diversos autores referem a existência de fatores de aspecto social e biológico com potencialidade para condicionar a atividade física. Segundo Wold \& Hendry 54, o maior envolvimento dos meninos pode ser explicado em parte por aspectos de natureza sócio-cultural. Enquanto os meninos eram desde as idades mais baixas orientados para atividades de âmbito laboral, as meninas eram direcionadas para a família e para atividades de expressão. Weinberg \& Gould 55 reforçam esta idéia ao relatarem que, desde o nascimento, as meninas e os meninos são tratados de forma diferente pelos adultos e pela sociedade, tendo os meninos uma maior permissão para explorar o seu ambiente físico. Estes autores salientam ainda que, durante décadas, a sociedade não atribuía ao sexo feminino o mesmo estatuto social do masculino visto não considerar aceitável a participação de meninas em atividades com elevadas exigências físicas e em que o contacto corporal estivesse presente, pois poderia comprometer a sua feminilidade.

Do mesmo modo, McKenzie et al. 56 destacam que a maior participação em atividades desportivas dos meninos poderá resultar do maior número de reforços positivos de incentivo à sua prática que recebem durante o período escolar e fora dele nos clubes e associações desportivas. Outras explicações avançadas para o menor envolvimento desportivo das meninas são as diferentes concepções do corpo, capacidades e atitudes necessárias à prática de atividades desportivas 57 . Do ponto de vista sócio-cultural, a concepção do corpo que está normalmente associada ao desporto não se enquadra muito bem nos modelos femininos atuais da corporalidade. Efetivamente, o corpo ideal feminino caracteriza-se pela graciosidade, elegância, beleza e relativa fragilidade, o que parece não se ajustar às imagens do corpo desportivo. Pelo contrário, um corpo musculado, forte, resistente e "agressivo", característico 
do sexo masculino, parece estar associado aos ideais de sucesso da grande maioria das modalidades desportivas. Esses fatores levam a que as meninas coloquem algumas reservas acerca da possibilidade da prática desportiva intensa afetar a sua feminilidade 55 .

Para além de fatores de âmbito social, as diferenças entre sexos podem ser igualmente devidas a fatores biológicos. Bar-Or \& Rowland 58 relatam que o aumento da gordura corporal, o alargamento pélvico, bem como o desconforto do período menstrual poderão ser razões suficientes para o menor envolvimento em atividades físicas por parte das meninas.

Em suma, ainda que exista alguma evidência da maior participação dos meninos em atividades físicas, especialmente as de âmbito desportivo e de intensidade vigorosa, e das meninas em atividades de lazer e de baixa intensidade, de uma forma geral observa-se um maior envolvimento do sexo masculino em atividades físicas. A presença deste dimorfismo sexual deve ser seriamente considerada pelos gestores de programas de intervenção no amplo universo da Saúde Pública, particularmente a fim de eliminar preconceitos sociais sobre o papel da mulher na prática de atividade física generalizada, culturalmente referenciada e prestigiante do ponto de vista pessoal.

\section{Estatuto sócio-econômico}

O estatuto sócio-econômico é outro dos determinantes que mais tem sido referenciado na literatura como modulador da prática de atividade física. Os resultados, entretanto, são pouco consensuais e não permitem identificar com clareza o sentido e a magnitude da associação entre o estatuto sócio-econômico e a atividade física. De fato, é possível encontrar estudos que revelam a existência de uma associação positiva entre o estatuto sócio-econômico e a atividade física $26,30,34,36,37,38,46,47,50,52,59$ e outros em que essa associação é negativa 5,60,61 ou inexistente $62,63,64$. A principal razão para esta divergência nos resultados poderá ser encontrada eventualmente no modo como o estatuto sócio-econômico tem sido avaliado. Na literatura, observamos estudos que avaliaram o estatuto sócio-econômico pelo rendimento familiar, pela formação acadêmica dos elementos constituintes do agregado familiar e pela atividade ocupacional desenvolvida por cada um dos membros da família.

Apesar de alguma controvérsia nos resultados, a generalidade das pesquisas parece evidenciar que a participação em atividades físicas apresenta alguma desigualdade social e econômica, isto é, adolescentes com um elevado estatuto sócio-econômico parecem estar mais envolvidos em atividades físicas do que adolescentes com um baixo estatuto. A literatura ressalta que a posição social e econômica de qualquer agregado familiar tende a estar positivamente associada à educação e à formação dos seus membros. Isso significará, por exemplo, que os adolescentes cujos pais se encontram em elevadas posições sociais estão mais propensos a ter uma educação e uma formação que possam resultar, na idade adulta, numa elevada posição social.

Por outro lado, também é sabido que o fato de os adolescentes passarem uma grande porcentagem do seu tempo diário na escola obriga a que muita da sua possível participação em atividade física tenha que ser realizada fora dos períodos escolares. Contudo, para que isso possa acontecer, terão de ter suporte social e econômico que lhes permita aceder a essas atividades que normalmente decorrem em organizações desportivas (clubes, associações, ginásios). Naturalmente que o acesso a essas organizações exige, entre outras coisas, que esses adolescentes tenham de se deslocar, de adquirir o equipamento necessário à sua prática e o pagamento de uma taxa de inscrição/manutenção. Segundo Sallis et al. 65, o fato de os pais terem a possibilidade de transportar os seus filhos para a prática de atividade física após a escola, e de pagar aulas ou treinos em organizações desportivas são aspectos que estão positivamente correlacionados com esse comportamento. De igual modo, White \& Coakley 66 documentaram algumas das restrições por que passam os adolescentes que vivem em famílias de baixo rendimento econômico que, apesar de estarem igualmente interessados na prática de atividades físicas, encontram dificuldade no pagamento de taxas de inscrição, na aquisição de material e equipamento específico e nos custos de transporte.

Em resumo, apesar de os resultados não serem suficientemente esclarecedores, os adolescentes de baixo estatuto sócio-econômico estão aparentemente em desvantagem no que se refere à participação em algumas formas de atividade física, especialmente as que se dão em clubes ou associações desportivas privadas. Políticas sociais de inclusão e de promoção generalizada de atividades físicas de toda a população gratuitamente deve ser uma prioridade tanto em países desenvolvidos quanto em desenvolvimento.

\section{Determinantes sócio-culturais}

O suporte social emerge como um importante e consistente determinante da atividade física de adolescentes. Dos diversos determinantes sócio-culturais mais referenciados na literatura, 
somente abordaremos a influência da família (progenitores e irmãos), dos pares e do professor de educação física na atividade física de adolescentes.

\section{Família e os pares}

A família tem sido identificada como uma das instituições tradicionais de educação que maior influência revela no envolvimento e participação de crianças e adolescentes em comportamentos saudáveis, existindo uma associação estreita entre a atividade desenvolvida pelos progenitores e a dos seus descendentes 43 . Segundo Duncan et al. 67, muitos dos comportamentos saudáveis são iniciados e mantidos no contexto familiar, podendo a família (pais e irmãos) ser considerada como um dos principais fatores de âmbito social capaz de influenciar os comportamentos dos adolescentes. O reconhecimento da importância que a família tem nos comportamentos evidenciados pelos adolescentes tem levado muitos investigadores à realização de estudos sobre a agregação familiar nos hábitos de atividade física (Tabela 4).
Da Tabela 4 facilmente se constata a existência de alguma variabilidade no sentido da associação entre a atividade física dos progenitores e a dos seus descendentes. Enquanto os resultados de alguns estudos mostram uma associação positiva, existem outros em que essa associação não se verifica. Apesar desta variação, a grande maioria das pesquisas (21/22 estudos) identifica a existência de agregação familiar nos hábitos de atividade física de adolescentes, mostrando que progenitores fisicamente ativos tendem a ter descendentes igualmente ativos.

Para reforçar esses resultados, parece-nos importante destacar dois estudos internacionalmente referenciados 68,69 que apenas não foram citados neste trabalho por não cumprirem todos os critérios definidos para a presente análise. No estudo de Moore et al. ${ }^{68}$, conduzido em 100 crianças dos 4 aos 7 anos de idade e respectivos progenitores, foi evidente que, quando o pai é fisicamente ativo, a propensão para a criança ser ativa é 3,4 vezes superior à de uma criança cujo pai é inativo. Por outro lado, quando a mãe é fisicamente ativa, a sua propensão é 2 vezes superior. Finalmente, quando os dois progenitores

Estudos sobre agregação familiar nos hábitos de atividade física de adolescentes.

\begin{tabular}{|c|c|c|c|c|c|c|}
\hline \multirow[t]{2}{*}{ Autor } & \multirow[t]{2}{*}{ Ano de realização } & \multirow[t]{2}{*}{ País } & \multicolumn{2}{|c|}{ Pai } & \multicolumn{2}{|c|}{ Mãe } \\
\hline & & & Filhos & Filhas & Filhos & Filhas \\
\hline Andersen \& Wold 75 & 1992 & Noruega & + & + & + & + \\
\hline Cleland et al. 85 & 2005 & Austrália & + & + & + & + \\
\hline Duncan et al. 67 & 2005 & Estados Unidos & 0 & 0 & 0 & 0 \\
\hline Fogelholm et al. 86 & 1999 & Finlândia & + & + & + & + \\
\hline Gottlieb \& Chen 87 & 1985 & Estados Unidos & + & + & + & + \\
\hline Gregson \& Colley 88 & 1986 & Inglaterra & 0 & + & 0 & + \\
\hline O'Loughlin et al. 26 & 1999 & Canadá & + & + & + & + \\
\hline Pereira 28 & 1999 & Portugal & ND & + & ND & + \\
\hline Pizarro \& Sherrill 77 & 1991 & Costa Rica & + & 0 & + & + \\
\hline Raudsepp \& Viira 72 & 2000 & Estônia & + & + & 0 & 0 \\
\hline Raudsepp \& Viira 73 & 2000 & Estônia & + & + & + & + \\
\hline Rossow \& Rise 43 & 1994 & Noruega & + & + & 0 & 0 \\
\hline Seabra 38 & 2004 & Portugal & + & + & $+/ 0$ & + \\
\hline Shropshire \& Carroll 63 & 1997 & Inglaterra & + & + & 0 & 0 \\
\hline Stucky-Ropp \& DiLorenzo 78 & 1993 & Estados Unidos & 0 & 0 & + & + \\
\hline Surís \& Parera 29 & 2005 & Espanha & + & + & + & + \\
\hline Vilhjalmsson \& Thorlindsson 49 & 1998 & Islândia & + & + & 0 & 0 \\
\hline Vilhjalmsson \& Kristjansdottir 30 & 2003 & Islândia & + & + & 0 & 0 \\
\hline Wagner et al. 50 & 2002 & França & 0 & 0 & + & + \\
\hline Wold \& Andersen 74 & 1992 & Países europeus & + & + & + & + \\
\hline Yang et al. 64 & 1996 & Finlândia & + & + & 0 & + \\
\hline Zakarian et al. 32 & 1994 & Estados Unidos & 0 & + & 0 & + \\
\hline
\end{tabular}

+: associação positiva; 0: associação inexistente; ND: não determinada; +/0: associação positiva no ensino básico e inexistente no ensino secundário. 
são fisicamente ativos, tal propensão é 7,2 vezes superior à de outras crianças cujos progenitores sejam fisicamente inativos. Na pesquisa de Freedson \& Evenson 69 , realizada em 30 crianças dos 5 aos 9 anos de idade e seus progenitores, verificou-se a existência de uma relação de forte magnitude entre o número de progenitores ativos e os níveis de atividade física evidenciados por essas crianças. Quando os pais eram muito ativos, aproximadamente $93 \%-97 \%$ das crianças também eram muito ativas.

A Tabela 4 mostra que a influência do pai e/ou da mãe não é consensual, sendo possível observar estudos que mostram uma associação mais significativa entre os níveis de atividade física da mãe e das filhas e entre os do pai e o dos filhos e outros em que se registra uma associação mais significativa de um dos progenitores relativamente ao outro (pai e ambos os filhos: 15/22 estudos; mãe e ambos os filhos: 11/22 estudos). Todavia, independentemente do sexo dos progenitores e dos seus descendentes, a participação em atividades físicas dos progenitores parece ter um impacto substancial no envolvimento, interesse e participação em atividades físicas dos seus descendentes.

$\mathrm{Na}$ literatura encontram-se descritos diferentes mecanismos pelos quais a família poderá promover os hábitos de atividade física dos adolescentes. Segundo Taylor et al. 70, os progenitores podem promover ou limitar a atividade física dos seus descendentes de uma forma direta e/ou indireta. Por um lado, têm a possibilidade de condicionar o acesso a equipamentos e a envolvimentos que facilitam a prática de atividade física; por outro, podem funcionar como modelos de participação nessas atividades.

A modelação é um conceito central na teoria da aprendizagem social que considera que os seres humanos podem aprender mediante observação dos comportamentos dos outros 71. A aplicação desse conceito ao domínio da atividade física resulta na hipótese de que os adolescentes cujos pais participam em atividade física têm maior probabilidade de serem igualmente ativas. Parece claro que as famílias e muito particularmente os pais possuem uma grande responsabilidade nos hábitos e comportamentos evidenciados pelos seus descendentes, visto funcionarem como modelos de comportamento apropriados, assim como por constituírem a principal fonte de reforço e incentivo à aquisição de um determinado comportamento.

A generalidade dos estudos $30,49,72,73,74$ tem identificado uma associação positiva entre a atividade física dos irmãos. A pesquisa de Wold \& Andersen 74 , realizada pela OMS em diferentes países europeus, sugere que entre os 11 e os 15 anos de idade, os valores de atividade física estão significativamente associados entre irmãos. Resultados semelhantes foram encontrados por Vilhjalmsson \& Thorlindsson 49 e Raudsepp \& Viira 72 . Nesses estudos, independentemente do sexo, o irmão mais velho influenciava significativamente a atividade física dos seus irmãos mais novos. Desse modo, ter irmãos mais velhos, que revelem um estilo de vida ativo, é possibilitar que estes funcionem como modelos positivos na aquisição de hábitos de atividades físicas por parte daqueles.

A influência dos pares na atividade física dos adolescentes está menos bem investigada do que a influência da família. Mas os estudos realizados mostram de forma consistente que os adolescentes que são fisicamente ativos tendem a ter amigos igualmente ativos 28,30,32,38,49,67,72,74,75,76,77,78.

De acordo com Wold \& Hendry 54 , a influência dos pares pode ser realizada da seguinte maneira: (1) os adolescentes influenciam-se mutuamente no iniciar de uma atividade; (2) um adolescente pode iniciar-se numa atividade pelo fato do seu melhor amigo ser ativo; e (3) relações de amizade são estabelecidas entre adolescentes que estão envolvidos em atividades físicas e desportivas. De igual modo, Duncan et al. 67 referem que o suporte social dos pares para a prática de atividades físicas de adolescentes pode ser observado na integração social (quando participam conjuntamente na atividade) e em aspectos emocionais (encorajamento) e instrumentais (partilha de equipamento e transporte)

Na literatura consultada, é freqüente a necessidade dos investigadores compreenderem se a magnitude da influência parental e/ou dos pares na prática de atividades físicas, tende a ser semelhante nas diferentes fases de crescimento e desenvolvimento dos adolescentes.

A família é consensualmente considerada como o primeiro e o principal agente de socialização na transmissão de comportamentos e estilos de vida ativos. Vários autores 9,67 reforçam essa idéia ao citarem que é durante a primeira década de vida que a influência da família na atividade física melhor se expressa por meio da modelação de interesses e habilidades, no reforço de comportamentos e no possibilitar do acesso às atividades.

A entrada na adolescência tende a ser um período crítico de afirmação, de autonomia e de independência em que os jovens tendem a "ignorar" os conselhos, os valores e as atitudes dos seus pais, dando provavelmente mais relevância aos hábitos e comportamentos dos seus pares ou de outros agentes socialmente relevantes. Rossow \& Rise 43 destacam que a adolescência é um período em que há uma reorientação das influências 
parentais para os pares, podendo naturalmente ocorrer uma alteração nos comportamentos saudáveis evidenciados pelos adolescentes.

Apesar dos resultados diversos, alguns estudos têm sido desenvolvidos com o propósito de identificar se a influência da família e dos pares permanece constante ao longo dos diferentes períodos de desenvolvimento de uma criança e de um jovem. Greendorfer 79, utilizando uma amostra de atletas do sexo feminino, constatou uma diminuição da influência familiar com o evoluir da idade. Enquanto na infância a família tinha uma enorme importância no envolvimento desportivo dessas jovens, na adolescência tal influência deixou de existir. Relativamente à influência dos pares, foi possível verificar que se manteve constante ao longo dos diferentes estádios de desenvolvimento. Resultados semelhantes foram igualmente observados no estudo de Andújar \& Piéron ${ }^{44}$, pois foi indiscutível uma diminuição da influência dos progenitores nos hábitos de atividade física dos descendentes à medida que a escolaridade aumentava.

Em suma, a família representa um determinante altamente significativo dos hábitos de atividade física de adolescentes. Adolescentes filhos de progenitores ativos têm maiores chances de também serem ativos. Os resultados mostram que os adolescentes que participam em atividades físicas tendem a ter pares igualmente ativos. Com o decorrer da idade a influência da família tende a diminuir, contrariamente à dos pares que costuma aumentar.

\section{Professor de educação física}

É opinião generalizada que a escola e muito particularmente a disciplina de educação física e o seu professor deverão ser determinantes importantes na promoção e desenvolvimento de estilos de vida ativos. Vilhjalmsson \& Thorlindsson 49 reforçam esta idéia ao alegarem que a escola, por intermédio das experiências positivas que a disciplina de educação física vai colocando, tem uma enorme contribuição nos hábitos de atividade física dos adolescentes.

Na literatura disponível, podemos encontrar alguns estudos que procuraram identificar a magnitude e o sentido da influência do professor de educação física na atividade física de adolescentes $28,30,38,49,65,76,77,79,80,81$. Os resultados são pouco consensuais e não permitem retirar conclusões esclarecedoras acerca da possível influência que o mestre poderá ter na atividade física de seus alunos. Esses dados parecem ser preocupantes, uma vez que seria esperado que o professor de educação física estivesse positivamente associado ao desenvolvimento físico e desporti- vo dos seus alunos e à aquisição de comportamentos e estilos de vida ativos. A preocupação torna-se ainda mais acentuada pelo fato de existir uma certa unanimidade quanto à diminuição da atividade física com o decorrer da idade, especialmente durante o período da adolescência; e por se saber que os adolescentes passam uma expressiva porcentagem do seu tempo diário na escola a participar de atividades de natureza letiva e não letiva. Estes dois aspectos têm levado os investigadores a considerar que é muito provável que o declínio dos níveis de atividade física tenha o seu início nas instituições escolares.

Uma outra razão invocada para tal diminuição dos níveis de atividade física dos adolescentes com o avanço da idade pode ser a elevada insatisfação e desinteresse que se registrava diante da disciplina de educação física e aos conteúdos programáticos que são lecionados. Godin \& Shephard 80 referem que o período crítico do desenvolvimento da insatisfação relativamente aos programas de educação física é na transição do 8o para o 9o ano de escolaridade. Os jovens

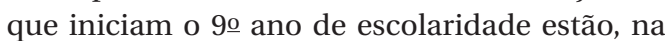
maior parte das vezes, insatisfeitos com a oferta desportiva, com o nível de competição e divertimento existente nas aulas de educação física. Sallis et al. 65 , numa pesquisa realizada com 1.504 adolescentes do 4o ao 12o ano de escolaridade salientam os mesmos aspectos e reforçam a enorme importância e o impacto que o prazer e o divertimento na disciplina de educação física poderá ter nos hábitos de atividade física dos adolescentes.

Assim, fica clara a necessidade de se repensar o papel interventivo dos programas da disciplina de educação física, nomeadamente alguma alteração dos objetivos, das matérias e conteúdos a ensinar, de maneira a conseguir manter altos níveis de participação, motivação e prazer pela prática de atividade física. Daqui que estejamos de acordo com a posição de Greenwood-Parr \& Oslin 82 quando afirmam que os estudantes terão maior propensão para se envolverem em atividades físicas se as aulas de educação física lhes proporcionarem experiências de autodeterminação e sensações de competência nas suas próprias capacidades. Por outro lado, é posição destes autores que semelhante exposição a diversas atividades físicas poderá ser o ponto de partida para que os adolescentes se tornem adultos ativos.

Para além da disciplina de educação física, McKenzie et al. 83 realçam a enorme importância do professor de educação física na aquisição e manutenção de hábitos de atividade física. $\mathrm{Na}$ opinião destes autores, este grupo social é aquele que no interior da comunidade escolar se encontra, teoricamente, melhor preparado para pro- 
mover a prática de atividades físicas no interior e no exterior da escola, nomeadamente por causa da formação de adolescentes que sejam independentes na sua prática e por possibilitar mais informação sobre iniciativas existentes na comunidade. Afora essas possibilidades, o professor de educação física poderá, no interior da escola e para além das aulas que leciona, ter um importante papel na promoção da atividade física por meio da criação e da organização de atividades antes do início das atividades letivas, nos seus intervalos e no seu término 84.

Em suma, apesar da ausência de unanimidade, a escola, a disciplina de educação física e o seu professor parecem desempenhar papéis importantes na aquisição e na manutenção de estilos de vida saudáveis por parte dos educandos. Os adolescentes podem chegar a passar boa parte do seu tempo diário na escola, sendo esse um espaço enorme de oportunidades para a implantação de programas variados e motivadores de atividades físicas.

\section{Conclusões}

Deste quadro genérico de resultados encontrados nos estudos consultados, destacamos as seguintes conclusões: (1) a idade parece ser um determinante importante dos níveis de atividade física dos adolescentes, visto que, à medida que aumenta, a atividade física tem tendência a diminuir; (2) o sexo (gênero) é um fator determinante da atividade física de adolescentes, sendo evidente que o sexo masculino está mais envolvido em atividades físicas que os seus pares do sexo oposto; (3) o estatuto sócio-econômico elevado termina por ser um fator protetor do risco de inatividade física em meninos e meninas; (4) o envolvimento e a participação da família e dos pares em atividades físicas parecem estar positivamente associados à atividade física de adolescentes; (5) o professor de educação física parece não representar um fator propiciador nos níveis de atividade física.

Cabe-nos salientar o importante significado e o alcance daquilo que emerge das conclusões desta pesquisa, sobretudo na revelação do seu potencial no estabelecimento e implementação de programas variados de atividades físicas. Ressaltamos, assim, a necessidade de esses programas: (a) serem aplicados em adolescentes com idades compreendidas entre os 10 e os 18 anos, já que a atividade física tende a diminuir no decorrer desse intervalo etário; (b) terem um enfoque especial nas meninas por se encontrarem numa situação de risco mais elevada de inatividade, exigindo-se por isso o estabelecimento de programas mais interessantes e variados, dirigidos àquilo que as adolescentes esperam; (c) serem mais democráticos na sua acessibilidade e não exigirem grande suporte de pessoal especializado, de material e equipamento desportivo e sobremodo financeiro; (d) envolverem uma rede alargada de fatores de âmbito sócio-cultural com capacidade para influenciar a atividade física de adolescentes e muito especialmente a família e os pares; (e) consciencializarem o professor de educação física de que é, na comunidade escolar, o elemento com maior preponderância na promoção e criação de hábitos de atividades físicas junto aos seus alunos. 


\section{Resumo}

A atividade física é um comportamento importante na promoção de saúde e na prevenção de doenças. Para que se desenvolvam programas eficazes no incentivo à prática de atividade física em adolescentes, torna-se necessário que se identifiquem os fatores que a determinam. Este estudo pretende rever alguns dos aspectos do estado atual do conhecimento acerca da influência de determinantes demográfico-biológicos (idade, sexo, estatuto sócio-econômico) e sócio-culturais (família, pares e professor de educação física) na atividade física de adolescentes. Nesta revisão apenas foram incluidos estudos efetuados com amostras superiores a 100 adolescentes com idades entre os 10 e os 18 anos, que tenham adotado delineamentos de pesquisa transversal e que tenham utilizado questionários. Os principais resultados e conclusões foram que: a idade parece estar negativamente associada à atividade física; o sexo masculino tende a estar mais envolvido nessas atividades; o estatuto sócio-econômico elevado parece ser um fator protetor do risco de inatividade física; a participação da família e dos pares em atividades físicas parece estar positivamente associada às atividades por parte dos adolescentes; o professor de educação física parece não representar um fator propiciador da atividade física.

Atividade Motora; Adolescente; Comportamento do Adolescente

\section{Referências}

1. World Health Organization. The World Health Report: reducing risks, promoting healthy life. Geneva: World Health Organization; 2002.

2. Sjostrom M, Oja P, Hagstromer M, Smith BJ, Bauman A. Health-enhancing physical activity across European Union countries: the Eurobarometer study. J Public Health 2006; 14:291-300.

3. Hallal PC, Samuel SC, Bastos JP, Reichert FF, Siqueira FV, Azevedo MR. Evolução da pesquisa epidemiológica em atividade física no Brasil: revisão sistemática. Rev Saúde Pública 2007; 41: 453-60.

4. World Health Organization. Young people's health in context. Health behaviour in school-aged children (HBSC) study: international report from 2001/2002 survey. Geneva:World Health Organization; 2004.

\section{Colaboradores}

A. F. Seabra contribuiu na concepção e estruturação do estudo; seleção e análise dos artigos de interesse e redação do texto. D. M. Mendonça, M. A. Thomis, L. A. Anjos e J. A. Maia contribuíram na concepção, estruturação e revisão do conteúdo do artigo.

\section{Agradecimentos}

Aos revisores anônimos cujos pareceres melhoraram a qualidade do texto. Trabalho financiado pela Fundação para a Ciência e Tecnologia (FCT) de Portugal com a referência SFRH/BD/20166/2004. Luiz A. Anjos recebeu bolsa de produtividade em pesquisa do Conselho Nacional de Desenvolvimento Científico e Tecnológico (CNPq, processo 302952/03-9).
5. Hallal PC, Bertoldi AD, Gonçalves H ,Victora CG. Prevalência de sedentarismo e fatores associados em adolescentes de 10-12 anos de idade. Cad Saúde Pública 2006; 22:1277-87.

6. Strong WB, Malina RM, Blimkie CJ, Daniels SR, Dishman RK, Gutin B, et al. Evidence based physical activity for school-age youth. J Pediatr 2005; 146:732-7.

7. Hallal PC, Victora CG, Azevedo MR, Wells JC. Adolescent physical activity and health: a systematic review. Sports Med 2006; 36:1019-30.

8. Rowland T. Physical activity, fitness, and children. In: Bouchard C, Blair SNH, Haskell WL, editors. Physical activity and health. Champaign: Human Kinetics; 2006. p. 259-70. 
9. Dishman RK, Sallis JF, Orenstein D. The determinants of physical activity and exercise. Public Health Rep 1985; 100:158-72.

10. Bouchard C, Shephard R, Stephens T. Physical activity, fitness and health: International Proceedings and Consensus Statement. Champaign: $\mathrm{Hu}-$ man Kinetics; 1994.

11. Sallis JF. A North American perspective on physical activity. In: Cameron J, Bar-Or O, editors. New horizonts in pediatric exercise science. Champaign: Human Kinetics; 1995. p. 221-34.

12. Bauman AE, Sallis JF, Dzewaltowski DA, Owen N. Toward a better understanding of the influences on physical activity: the role of determinants, correlates, causal variables, mediators, moderators, and confounders. Am J Prev Med 2002; 23:5-14.

13. Bouchard C, Malina R, Pérusse L. Genetics of fitness and physical performance. Champaign: Human Kinetics; 1997.

14. Caspersen CJ, Nixon PA, DuRant RH. Physical activity epidemiology applied to children and adolescents. Exerc Sport Sci Rev 1998; 26:341-403.

15. Buckworth J, Dishman RK. Determinants of exercise and physical activity. In: Bahrke M, editor. Exercise psychology. Champaign: Human Kinetics; 2002. p. 191-209.

16. Sallis JF, Prochaska JJ, Taylor WC. A review of correlates of physical activity of children and adolescents. Med Sci Sports Exerc 2000; 32:963-75.

17. Caspersen CJ, Powell KE, Christenson GM. Physical activity, exercise, and physical fitness: definitions and distinctions for health-related research. Public Health Rep 1985; 100:126-31.

18. Dishman RK, Washburn RA, Heath GW. Physical activity epidemiology. Champaign: Human Kinetics; 2004.

19. van Mechelen W, Mellenbergh GJ. Problems and solutions in longitudinal research: from theory to practice. Int J Sports Med 1997; 18 Suppl 3:S238-45.

20. LaPorte RE, Montoye HJ, Caspersen CJ. Assessment of physical activity in epidemiologic research: problems and prospects. Public Health Rep 1985; 100:131-46.

21. Montoye HJ, Kemper HC, Saris WH ,Washburn RA. Measuring physical activity and energy expenditure. Champaign: Human Kinetics; 1996

22. Welk GJ. Physical activity assessments for healthrelated research. Champaign: Human Kinetics; 2002.

23. Aaron DJ, Jekal YS, LaPorte RE. Epidemiology of physical activity from adolescence to young adulthood. World Rev Nutr Diet 2005; 94:36-41.

24. Carron A, Hausenblas H, Estabrooks P. Individual correlates of physical activity. In: Dorwick T, editor. The psychology of physical activity. New York: McGraw-Hill; 2003. p. 85-93.

25. Kristjansdottir G, Vilhjalmsson R. Sociodemographic differences in patterns of sedentary and physically active behavior in older children and adolescents. Acta Paediatr 2001; 90:429-35.

26. O'Loughlin J, Paradis G, Kishchuk N, Barnett T, Renaud L. Prevalence and correlates of physical activity behaviors among elementary schoolchildren in multiethnic, low income, inner-city neighborhoods in Montreal, Canada. Ann Epidemiol 1999; 9:397-407.
27. Pate RR, Trost SG, Felton G, Ward DS, Dowda M, Saunders R. Correlates of physical activity behavior in rural youth. Res Q Exerc Sport 1997; 68:241-8.

28. Pereira P. Influência parental e outros determinantes nos níveis de actividade física: um estudo em jovens do sexo feminino dos 12 aos 19 anos [Dissertação de Mestrado]. Porto: Faculdade de Ciências do Desporto e de Educação Física, Universidade do Porto; 1999.

29. Surís J, Parera N. Don't stop, don't stop: physical activity and adolescence. Int J Adolesc Med Health $2005 ; 17: 67-80$

30. Vilhjalmsson R, Kristjansdottir G. Gender differences in physical activity in older children and adolescents: the central role of organized sport. Soc Sci Med 2003; 56:363-74.

31. Wolf AM, Gortmaker SL, Cheung L, Gray HM, Herzog DB, Colditz GA. Activity, inactivity, and obesity: racial, ethnic, and age differences among schoolgirls. Am J Public Health 1993; 83:1625-7.

32. Zakarian JM, Hovell MF, Hofstetter CR, Sallis JF Keating KJ. Correlates of vigorous exercise in a predominantly low SES and minority high school population. Prev Med 1994; 23:314-21.

33. Myers L, Strikmiller PK, Webber LS, Berenson GS Physical and sedentary activity in school children grades 5-8: the Bogalusa Heart Study. Med Sci Sports Exerc 1996; 28:852-9.

34. Woodfield L, Duncan M, Al-Nakeeb Y, Nevill A, Jenkins C. Sex, ethnic and socio-economic differences in children's physical activity. Pediatr Exerc Sci 2002; 14:277-85.

35. Grunbaum JA, Kann L, Kinchen S, Ross J, Hawkins J, Lowry R, et al. Youth risk behavior surveillance United States, 2003 (Abridged). J Sch Health 2004; 74:307-24.

36. Lasheras L, Aznar S, Merino B, Lopez EG. Factors associated with physical activity among Spanish youth through the National Health Survey. Prev Med 2001; 32:455-64.

37. Schmitz KH, Lytle LA, Phillips GA, Murray DM, Birnbaum AS, Kubik MY. Psychosocial correlates of physical activity and sedentary leisure habits in young adolescents: the Teens Eating for Energy and Nutrition at School Study. Prev Med 2002; 34:266-78.

38. Seabra A. Influência de determinantes demográfico-biológicos e sócio-culturais nos níveis de actividade física de crianças e jovens [Dissertação de Mestrado]. Porto: Faculdade de Medicina, Universidade do Porto; 2004

39. Rowland TW. The biological basis of physical activity. Med Sci Sports Exerc 1998; 30:392-9.

40. Thorburn AW, Proietto J. Biological determinants of spontaneous physical activity. Obes Rev 2000; 1:87-94.

41. Ingram DK. Age-related decline in physical activity: generalization to nonhumans. Med Sci Sports Exerc 2000; 32:1623-9.

42. Sallis JF, Owen N. Physical activity \& behavioral medicine. London: Sage Publications; 1999

43. Rossow I, Rise J. Concordance of parental and adolescent health behaviors. Soc Sci Med 1994; 38:85-92. 
44. Andújar A, Piéron M. La incidencia de la práctica físico-deportiva de los padres hacia sus hijos durante la infancia y la adolescencia. Apunts - Educación Física y Deportes 2000; 65:100-4.

45. Bungun T, Dowda M, Weston A, Trost S, Pate RR. Correlates of physical activity in male and female youth. Pediatr Exerc Sci 2000; 12:71-9.

46. Oehlschlaeger MHK, Pinheiro RT, Horta B, Gelatti C, San'Tana P. Prevalência e fatores associados ao sedentarismo em adolescentes de área urbana. Rev Saúde Pública 2004; 38:157-63.

47. Sallis JF, Zakarian JM, Hovell MF, Hofstetter CR. Ethnic, socioeconomic, and sex differences in physical activity among adolescents. J Clin Epidemiol 1996; 49:125-34.

48. Trost SG, Pate RR, Dowda M, Saunders R, Ward DS, Felton G. Gender differences in physical activity and determinants of physical activity in rural fifth grade children. J Sch Health 1996; 66:145-50.

49. Vilhjalmsson R, Thorlindsson T. Factors related to physical activity: a study of adolescents. Soc Sci Med 1998; 47:665-75.

50. Wagner A, Klein-Platat C, Haan M, Arveiler D, Shlienger J, Simon C. Relations entre niveau d'activité physique des collégiens et celui de leurs parents: associations avec le niveau socio-économique. Rev Epidemiol Sante Publique 2002; 50:74-5.

51. Levin S, Lowry R, Brown DR, Dietz WH. Physical activity and body mass index among US adolescents: youth risk behavior survey, 1999. Arch Pediatr Adolesc Med 2003; 157:816-20.

52. Duncan M, Woodfield L, Al-Nakeeb Y, Nevill A. The impact of socio-economic status on the physical activity levels of British secondary school children. European Journal of Physical Education 2002; 7:30-44.

53. Hovell MF, Sallis JF, Kolody B, McKenzie TL. Children's physical activity choices: a development analysis of gender, intensity levels, and time. Pediatr Exerc Sci 1999; 11:158-68.

54. Wold B, Hendry L. Social and environmental factors associated with physical activity in young people. In: Biddle S, Sallis JF, Cavill N, editors. Young and active? Young people and health-enhancing physical activity - evidence and implications. London: Health Education Authority; 1998. p. 119-32.

55. Weinberg R, Gould D. Gender issues in sport and exercise. In: Gisolfi C, Lamb D, editors. Foundation of sport and exercise psychology. Indianapolis: Benchmark Press; 1995. p. 495-513.

56. McKenzie TL, Sallis JF, Elder JP, Berry CC, Hoy PL, Nader PR, et al. Physical activity levels and prompts in young children at recess: a two-year study of a bi-ethnic sample. Res Q Exerc Sport 1997; 68:195-202.

57. Malina RM. Tracking of physical activity and physical fitness across the lifespan. Res Q Exerc Sport 1996; 67(3 Suppl):S48-57.

58. Bar-Or O, Rowland T. Pediatric exercise medicine - from physiologic principles to health care application. Champaign: Human Kinetics; 2004.

59. Gordon-Larsen P, McMurray RG, Popkin B. Determinants of adolescent physical activity and inactivity patterns. Pediatrics 2000; 105:1327-8.
60. Fuchs R, Powell KE, Semmer NK, Dwyer JH, Lippert P, Hoffmeister H. Patterns of physical activity among German adolescents: the Berlin-Bremen Study. Prev Med 1988; 17:746-63.

61. Harrell JS, Pearce PF, Markland ET, Wilson K, Bradley CB, McMurray RG. Assessing physical activity in adolescents: common activities of children in 6th-8th grades. J Am Acad Nurse Pract 2003; 15:170-8.

62. Mota J, Silva G. Adolescent's physical activity: association with socio-economic status and parental participation among a Portuguese sample. Sport Education Society 1999; 4:193-9.

63. Shropshire J, Carroll B. Family variables and children's physical activity: influence of parental exercise and socio-economic status. Sport Education Society 1997; 2:95-116.

64. Yang X, Telama R, Laakso L. Parents' physical activity, socioeconomic status and education as predictors of physical activity and sport among children and youths - a 12 year follow-up study. International Review for the Sociology of Sport 1996; 31:273-91.

65. Sallis JF, Alcaraz JE, McKenzie TL, Hovell MF. Predictors of change in children's physical activity over 20 months: variations by gender and level of adiposity. Am J Prev Med 1999; 16:222-9.

66. White A, Coakley J. Making decisions: the response of young people in the medway towns to the "ever thought of sport?" campaign. London: Greater London and South East Region Sports Council; 1986.

67. Duncan SC, Duncan TE, Strycker LA. Sources and types of social support in youth physical activity. Health Psychol 2005; 24:3-10.

68. Moore L, Lombardi D, White M, Campbell J, Oliveria S, Ellison C. Influence of parents' physical activity levels on activity levels of young children. J Pediatr 1991; 118:215-9.

69. Freedson P, Evenson S. Familial aggregation in physical activity Res Q Exerc Sport 1991; 62:384-9.

70. Taylor WC, Baranowski T, Sallis JF. Family determinants of childhood physical activity: a social cognitive model. In: Dishman RK, editor. Advances in exercise adherence. Champaign: Human Kinetics; 1994. p. 319-42.

71. Bandura A. Self-efficacy: toward a unifying theory of behavioral change. Psychol Rev 1977; 84:919-25.

72. Raudsepp L, Viira R. Sociocultural correlates of physical activity in adolescents. Pediatr Exerc Sci 2000; 12:51-60.

73. Raudsepp L, Viira R. Influence of parents' and siblings' physical activity on activity levels of adolescents. European Journal of Physical Education 2000; 5:169-78.

74. Wold B, Andersen N. Health promotion aspects of family and peer influences on sport participation. Int J Sport Psychol 1992; 23:343-59.

75. Andersen N, Wold B. Parental and peer influences on leisure-time physical activity in young adolescents. Res Q Exerc Sport 1992; 63:341-8.

76. Greendorfer S, Lewko J. Role of family members in sport socialization of children. Res Q Exerc Sport 1978; 49:146-53.

77. Pizarro A, Sherrill C. Correlates of active sport involvement for urban Costa Rica children. Percept Mot Skills 1991; 73:807-10. 
78. Stucky-Ropp RC, DiLorenzo TM. Determinants of exercise in children. Prev Med 1993; 22:880-9.

79. Greendorfer S. Role of socializing agents in female sport involvement. Res Q Exerc Sport 1977; 48:304-10.

80. Godin G, Shephard R. Psychosocial factors influencing intentions to exercise of young students from grades 7 to 9. Res Q Exerc Sport 1986; 57: 41-52.

81. Pratt M, Macera CA, Blanton C. Levels of physical activity and inactivity in children and adults in the United States: current evidence and research issues. Med Sci Sports Exerc 1999; 31(11 Suppl): S526-33.

82. Greenwood-Parr M, Oslin J. Promoting lifelong involvement through physical activity. Am J Health Educ 1998; 69:72-6.

83. McKenzie TL, Marshall SJ, Sallis JF, Conway TL. Student activity levels, lesson context, and teacher behavior during middle school physical education. Res Q Exerc Sport 2000; 71:249-59.
84. Scruggs PW, Beveridge SK, Watson DL. Increasing children's school time physical activity using structured fitness breaks. Pediatr Exerc Sci 2003; 15:159-69.

85. Cleland V, Venn A, Fryer J, Dwyer T, Blizzard L. Parental exercise is associated with Australian children's extracurricular sports participation and cardiorespiratory fitness: a cross-sectional study. Int J Behav Nutr Phys Act 2005; 2:1-9.

86. Fogelholm M, Nuutinen O, Pasanen M, Myohanen E, Saatela T. Parent-child relationship of physical activity patterns and obesity. Int J Obes Relat Metab Disord 1999; 23:1262-8.

87 Gottlieb NH, Chen MS. Sociocultural correlates of childhood sporting activities: their implications for heart health. Soc Sci Med 1985; 21:533-9.

88. Gregson J, Colley A. Concomitants of sport participation in male and female adolescents. Int J Sport Psychol 1986; 17:10-22.

89. Lindquist C, Reynolds K, Goran M. Sociocultural determinants of physical activity among children. Prev Med 1999; 29:305-12.

Recebido em 28/Nov/2006

Versão final reapresentada em 17/Ago/2007 Aprovado em 27/Ago/2007 